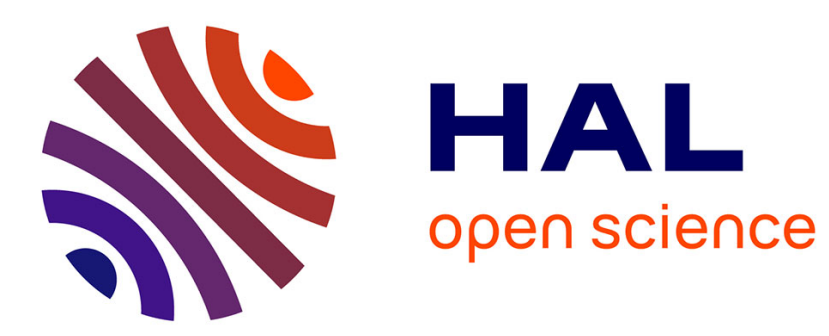

\title{
Les agoras grecques d'après les recherches récentes
}

Jean-Yves Marc

\section{To cite this version:}

Jean-Yves Marc. Les agoras grecques d'après les recherches récentes. Histoire de l'art, 1998, 42/43, pp.3-15. halshs-00005332

\section{HAL Id: halshs-00005332 \\ https://shs.hal.science/halshs-00005332}

Submitted on 8 Nov 2005

HAL is a multi-disciplinary open access archive for the deposit and dissemination of scientific research documents, whether they are published or not. The documents may come from teaching and research institutions in France or abroad, or from public or private research centers.
L'archive ouverte pluridisciplinaire HAL, est destinée au dépôt et à la diffusion de documents scientifiques de niveau recherche, publiés ou non, émanant des établissements d'enseignement et de recherche français ou étrangers, des laboratoires publics ou privés. 
Article paru dans « Architecture et décor» $N^{\circ}$ 42/43, octobre 1998 
Le mot " agora " désigne dans les villes grecques antiques le grand espace public réservé aux activités collectives, qu'elles soient politiques, religieuses, commerciales ou culturelles. L'agora constituait, au même titre que les sanctuaires, un des pôles autour desquels s'organisait l'espace urbain. Ces complexes, qui associèrent progressivement à une vaste aire ouverte un équipement monumental, sont un des éléments les plus originaux des villes de la Grèce antique, que l'on ne retrouve pas dans les civilisations urbaines plus anciennes et qui sont présents dans toutes les villes du monde grec. Apparus lors de la formation même des premiers véritables noyaux urbains, depuis le milieu du VIII ${ }^{\mathrm{e}}$ siècle, adaptés aux différentes fonctions qu'ils remplirent successivement et transformés au gré des modes architecturales ou urbanistiques, ils perdurèrent jusqu'à la fin de l'Antiquité : leur disparition, par destruction ou réoccupation des bâtiments et de l'espace, peut même être considérée comme le symptôme de la mort de la cité grecque.

Pourtant, l'agora, dont l'étude a commencé après celle des grands sanctuaires, n'est pas aussi connue qu'on a longtemps voulu le croire. Devenue le paradigme de l'urbanité comme vaste place monumentale, de la sociabilité comme lieu de promenade et d'échange, de la démocratie comme lieu de réunion du peuple et siège des magistratures, l'agora grecque a souffert d'une série de malentendus. L'ambiguïté du mot grec (du thème ager = rassembler) qui désigne, selon les périodes ou les contextes, les hommes qui se rassemblent, le lieu du rassemblement, l'assemblée en tant qu'institution politique, le discours à l'assemblée, le marché enfin, a pu entraîner une certaine confusion dans les études modernes, qui ont trop souvent cherché à retrouver dans les centres monumentaux dégagés par les fouilles l'ensemble des fonctions associées au mot agora, quelle que soit l'époque considérée. La polysémie du mot " agora " en grec ancien, illustration parmi d'autres de l'imprécision du vocabulaire technique antique, la multiplicité et les nombreuses variations des fonctions successives des centres monumentaux, l'extrême diversité des contextes historiques, qui couvrent l'ensemble de l'espace méditerranéen et même au-delà sur plus d'un millénaire, font que la réalité ne se laisse pas enfermer dans une formule simple, qu'elle soit terminologique ou architecturale. Sans parler de l'anachronisme qui consiste à désigner de ce mot les complexes palatiaux de l'Âge du Bronze, qu'il conviendrait d'abandonner sauf à traduire " agora " par place, il serait préférable de renoncer, y compris pour les époques historiques, à toute tentative de systématisation, du fait de l'inadéquation entre une dénomination unique et précise et une réalité diverse, voire contradictoire.

Par ailleurs, on a souvent tenté de faire correspondre les grandes étapes de son développement urbanistique et de son histoire architecturale avec les rythmes de l'histoire politique, recherchant en particulier un lien de cause à effet avec l'émergence ou le déclin des institutions civiques. On a surtout cité le cas athénien, bien que, dans ce domaine comme dans beaucoup d'autres, il apparaisse comme trop exceptionnel pour pouvoir servir de modèle : si l'agora d'Athènes est un des centres civiques sur lesquels on est le mieux renseigné aujourd'hui, grâce 
aux fouilles qui l'ont presque entièrement dégagée et à l'abondance des sources historiques, le réexamen critique dont elle fait l'objet depuis quelques années invite à une grande prudence.

Enfin, la documentation archéologique pose deux séries de problèmes difficiles à résoudre. D'une part, elle donne accès aux états les plus récents des agoras, correspondant aux époques hellénistique et romaine, et ne permet que rarement et partiellement de remonter aux phases les plus anciennes des centres civiques. Le danger est grand alors de raisonner sur un espace tel qu'il se présente au terme de son histoire, c'est-à-dire constitué et limité par des constructions : or ce n'est là que le résultat d'une évolution presque millénaire, qui a vu l'accumulation progressive d'édifices aux fonctions diverses et changeantes, mais aussi la disparition de nombreux autres. D'autre part, les constructions dans lesquelles on cherche à voir les sièges des différentes institutions de la cité ou les lieux d'échange sont très difficiles à interpréter : alors qu'un théâtre ou des thermes ne posent guère de problème d'identification, bien des constructions découvertes sur les agoras restent énigmatiques (édifices destinés à accueillir des tribunaux? un collège de magistrats ? un conseil ? des archives ?), bien des mentions épigraphiques d'édifices ne sont pas reconnues, y compris sur des centres monumentaux entièrement dégagés. C'est que les différents éléments qui composent la panoplie monumentale des cités grecques présentent rarement une correspondance assurée entre leur plan et leur fonction. Ces constructions civiques relèvent souvent d'enjeux symboliques qui déterminent - plus qu'on ne le pense - leur morphologie : le plan et la taille d'un édifice ne dépendent pas mécaniquement de nécessités fonctionnelles. L'archéologue, réduit à raisonner par analogie faute de sources épigraphiques, peut être conduit sur de fausses pistes.

Les travaux sur les agoras ont longtemps été dominés par la magistrale synthèse de Roland Martin en 1951. On ne discutera pas ici ses principales conclusions (reprises, précisées ou développées dans les nombreux travaux qu'il publia jusqu'à sa mort sur le même sujet), mais disons que, si les grands types qu'il a pu définir (agora des cités fondées sur un site vierge/agora de cités " évolutives", agora de cités commerçantes/agora de cités rurales, etc.) conservent une indéniable valeur heuristique, ils n'ont plus aujourd'hui la même netteté. Ce sont d'ailleurs souvent les travaux qu'il a suscités ou lancés lui-même, en particulier en Grande Grèce ou en Sicile, qui ont contribué à modifier notre approche des agoras.

Les nouvelles recherches s'articulent autour de plusieurs chantiers. Tout d'abord les fouilles qui ont récemment permis de dégager plusieurs centres monumentaux nouveaux et d'y atteindre parfois les états les plus anciens : Mégara Hyblaea, Sélinonte, Poseidonia, Métaponte pour les cités coloniales de l'ouest, Corinthe dans le Péloponnèse. Des villes de la Grèce du Nord, région considérée à tort comme marginale dans le monde grec, sont en cours d'exploration : Kassopé en Épire, Démétrias en Thessalie, Dion, Pella ou Thessalonique en Macédoine illustrent mieux qu'ailleurs les programmes monumentaux du début de l'époque hellénistique. En Asie Mineure, les grands sites de la côte comme Pergame, Milet ou Éphèse, et les fouilles récentes de cités de l'intérieur comme Aphrodisias ou Sagalassos, permettent d'élargir les recherches à la basse époque hellénistique ou à l'époque impériale. Même les agoras dégagées depuis longtemps, comme à Athènes, à Mégalopolis, à Messène ou à Thasos, connaissent un regain d'intérêt : nouvelles fouilles et réexamen des vestiges anciennement mis au jour permettent de reprendre des questions laissées sans réponse ou d'abandonner des restitutions admises trop rapidement. Enfin, les recherches menées aux confins du monde grec, à Aï Khanoum en Bactriane (Afghanistan) ou à Doura Europos sur les bords du Tigre, montrent comment est transposé dans ces villes un élément né dans des espaces urbains et des contextes sociopolitiques très différents.

À cet enrichissement considérable de la documentation archéologique, sont venus s'ajouter des changements de perspectives. Sans tomber dans l'hypercritique, on renonce aujourd'hui avec raison à solliciter trop fortement les sources écrites, en général très postérieures aux vestiges archéologiques avec lesquelles on les mettait en rapport. Les sources épigraphiques, qui concernent les périodes où les vestiges archéologiques sont les plus nombreux et les moins mal assurés, y ont gagné un regain d'intérêt. On reconsidère également les espaces monumentaux, en essayant de ne pas se laisser enfermer dans les limites léguées par les époques plus tardives: le passage du terrain vague à la place fermée a été très progressif et les grandes colonnades hellénistiques n'ont fait parfois que monumentaliser un secteur particulier du centre civique. Cette vision moins téléologique et beaucoup plus génétique de l'espace semble plus proche des réalités antiques.

4 LES AGORAS GRECQUES D'APRÈS LES RECHERCHES RÉCENTES 


\section{L'émergence d'un espace}

Si le mot agora apparaît dès les poèmes homériques, la réalité archéologique d'un grand espace clairement défini et équipé pour recevoir les assemblées politiques ou toute autre institution de la cité ne semble prendre forme qu' au VII ${ }^{\mathrm{e}}$ ou au VI ${ }^{\mathrm{e}}$ siècle av. J.-C. selon les cas. La cité idéale, représentée sur le bouclier d'Achille décrit dans l'Iliade, comprend une agora, définie comme un cercle sacré et munie de sièges en marbre (Il., XVIII, 503-4). On retrouve également un dispositif destiné à accueillir une assemblée sur l'agora d'Ithaque (Od., II, 14), sur celle des Phéaciens (Od., VI, 262-269) et, lorsque Alkinoos accueille Ulysse, il le fait venir sur l'agora où il l'invite à s'asseoir sur un siège en marbre (Od., VIII, 4-7). Le mot " agora " semble bien désigner alors un espace précis qui remplit un rôle politique (réunion d'assemblées ou de conseils, accueil des étrangers). Mais en dehors de ces occurrences, dont on ignore l'historicité, l'agora désigne plutôt dans la littérature grecque archaïque soit une réunion sans localisation précise, soit un marché. Il faut attendre les bornes retrouvées en bordure de l'agora d'Athènes ou le Code de Gortyne (col. X, 34-5 et XI, 12-4) au début du Ve siècle pour que le mot désigne également un emplacement précis à l'intérieur de la ville. Or à Thasos, vers 470 av. J.-C., un règlement de voirie qui concerne dans une de ses clauses le centre civique, n'emploie pas pour le désigner le mot " agora ", mais une périphrase qui suggère un centre défini par les édifices qui le bordent. Plus tard au cours du Ve siècle, le mot n'a pas encore acquis définitivement son sens de place publique à fonction politique, puisqu'il renvoie chez Hérodote à un marché (I, 153, 1-2).

Ce sont, pour le moment, les cités coloniales de Grande Grèce ou de Sicile qui nous renseignent le mieux sur les débuts de l'agora. Celle-ci semble d'abord consister en un espace libre, prévu dès l'implantation des colons, mais protégé par un sanctuaire et sans édifice civique particulier : il s'agit alors d'un dégagement défini dans le réseau des rues, dont l'intégrité serait protégée par la sacralité d'un autel, d'un temple ou d'une tombe de héros (herôon), et non par une fonction civique particulière. À Mégara Hyblaéa, cité fondée sur la côte est de la Sicile au milieu du VIII ${ }^{\mathrm{e}}$ siècle, le réseau viaire réserve dès le début un quadrilatère dont le plan trapézoïdal résulte de la rencontre, à cet endroit, de deux systèmes d'orientation différents. Si ce terrain ne fut jamais bâti, il ne reçut sa première parure monumentale qu'un siècle plus tard. Au milieu du VII ${ }^{\mathrm{e}}$ siècle, il s'étend en effet sur des parcelles voisines précédemment occupées par des maisons et accueille les premiers édifices sur ses bordures. Il s'agit surtout de l'herôon à l'ouest, d'un portique au nord et d'un temple au sud. Cet exemple, sans doute un des plus anciens mis au jour jusqu'à maintenant, montre que l'agora est au départ un espace reconnu comme bien commun par la communauté des habitants et protégé par la présence d'un ou plusieurs cultes : on ne peut naturellement exclure qu'il servait de lieu de rencontre, mais rien n'indique qu'à cette époque s'y déroulait une quelconque activité politique. Le terrain qui émerge alors à l'intérieur de l'espace bâti n'a pas encore la spécificité civique des agoras de l'époque classique.

La présence de lieux de culte se retrouve d'ailleurs dans de très nombreux exemples, qu'ils soient mythiques, comme l'agora des Phéaciens qui entoure le sanctuaire de Poséidon (Od., VI, 266-267), ou concrets comme à Cyrène, où trône dès le début du VI ${ }^{\mathrm{e}}$ siècle av. J. -C. " sur la poupe de l'agora " (Pindare, Pyth., V, 89-96) l'herôon du fondateur Battos, et comme à Thasos, où Glaukos, un des fondateurs de la colonie, avait, au moins depuis le début du ve siècle av. J.-C., son cénotaphe. Là, l'entrée de l'agora est même marquée vers 470 av. J.-C. par un passage monumental qui abritait une série de cultes importants, le passage des Théores. Cette absence de différenciation nette, au moins à l'origine, entre espace politique et espace religieux, repose sur le fait que, dans la plupart des cas, l'agora émerge d'un vaste espace dominé par un ou plusieurs sanctuaires. À Métaponte, où un gigantesque secteur fut réservé à l'intérieur de la ville, ce sont des temples qui y sont d'abord construits (fig. 1). L'espace " agora " ne prend une forme concrète qu'avec l'installation d'un nouveau sanctuaire au sud de la zone des temples : il s'agit du sanctuaire de Zeus, dont l'épiclèse agoraios désigne précisément le lieu. Prend forme alors au sein même de la grande aire sacrée une sorte d'agora de Zeus. Mais ce n'est que plus tard qu'une barrière ajourée limitera plus nettement les grands sanctuaires poliades et le centre civique. 


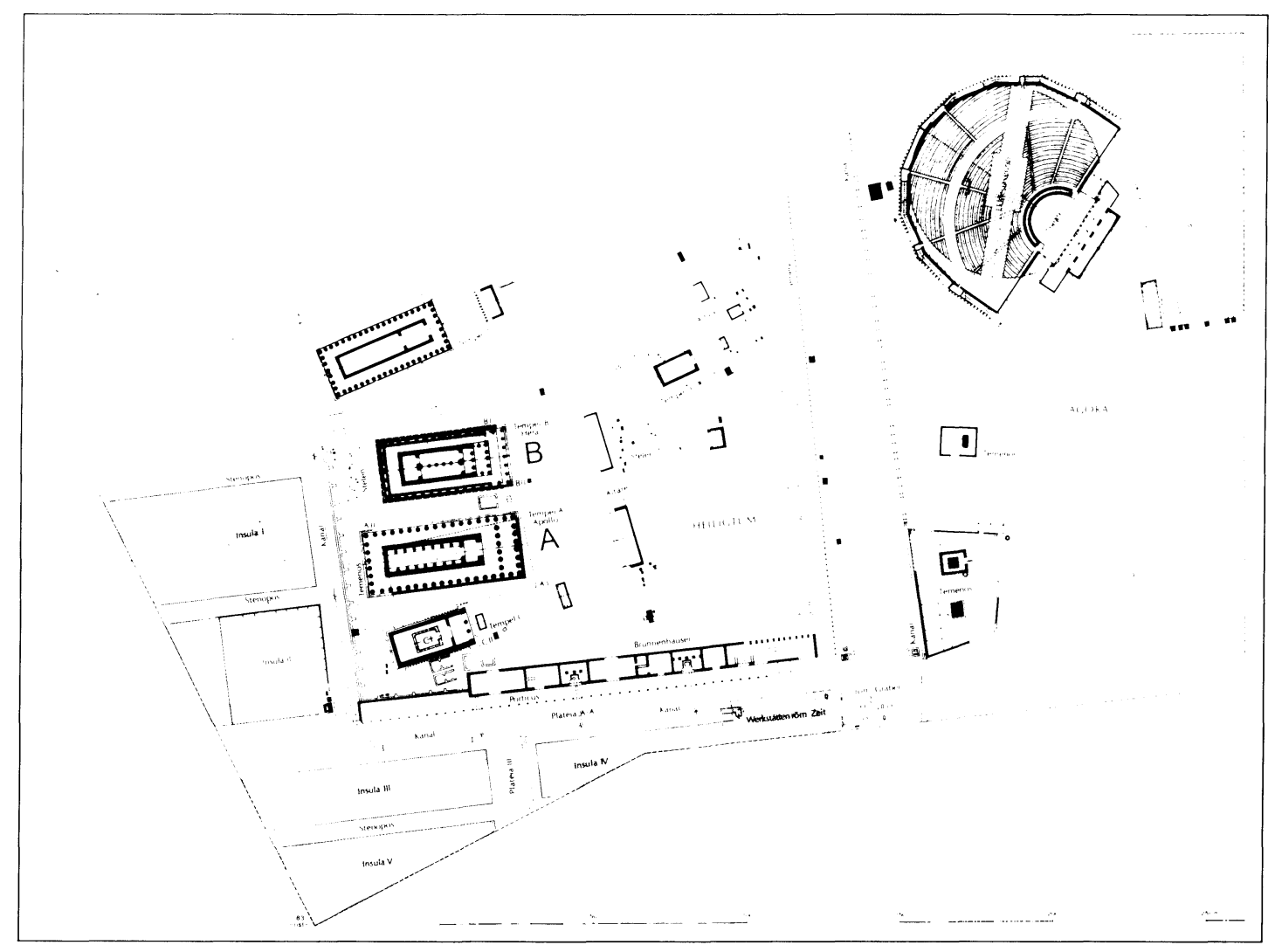

Fig. 1

Plan restitué de l'agora de Métaponte. Les édifices antérieurs au ve siècle sont : les temples $\mathrm{A}$ et $\mathrm{B}$,

le sanctuaire de Zeus Agoraios et l'ecclesiasterion. (D'après D. Mertens, Augusteische Architektur in Ephêsos, Vienne, 1974)

Les agoras conserveront toujours ce lien originel avec les cultes et les sanctuaires. Parfois même la différenciation ne sera jamais réalisée, comme à Olympie, où prytanée et bouleuterion furent construits, l'un dans l'Altis, l'autre à proximité immédiate; comme à Délos, où l'on trouve le prytanée de la cité dans le sanctuaire d'Apollon ou à sa proximité immédiate; comme à Thermos, où sanctuaire et agora sont complètement confondus. Ailleurs, les agoras continuent d'accueillir des manifestations religieuses : ainsi, sur l'Agora d'Athènes, les concours tragiques des Grandes Dionysies, jusqu'à la construction d'un théâtre sur les pentes sud de l'Acropole, ou la grande procession des Panathénées. À Argos, orchestra et dromos signalent également le lien de l'agora avec les fêtes et les concours liés aux manifestations religieuses essentielles à la cité. D'une manière générale, on est frappé par la quantité de sanctuaires, plus ou moins importants, qui se trouvaient sur les agoras jusqu'à l'époque impériale comprise. Il suffit de rappeler la description par Pausanias de l'agora de Mégalopolis en Arcadie : pas moins d'une dizaine de sanctuaires y sont mentionnés. Née dans la lignée des espaces sacrés, accueillant les manifestations religieuses, l'agora grecque ne fut cependant jamais, au moins à partir de l'époque classique, dominée par un temple : le temple de Zeus construit sur l'agora de Magnésie du Méandre est de taille extrêmement réduite; celui de Zeus Sôter, sur l'agora de Mégalopolis, est nettement plus grand, mais il semble comme écrasé par l'immensité de la place. Cette caractéristique constitue une différence notable entre les agoras grecques et les fora romains. Malgré un réel processus d'autonomie de l'espace " agora " par rapport aux cultes qui présidèrent à sa naissance, il n'est pas sûr qu'on puisse parler d'un mouvement de laïcisation. La concentration très progressive des édifices civiques ou destinés à abriter des activités commerciales est simplement venue enrichir la panoplie monumentale des places primitives : il n'y eut jamais de substitution pure et simple d'une fonction strictement civique ou collective à une fonction religieuse.

6 LES AGORAS GRECQUES D'APRÈS I.ES RECHERCHES RÉCENTES 


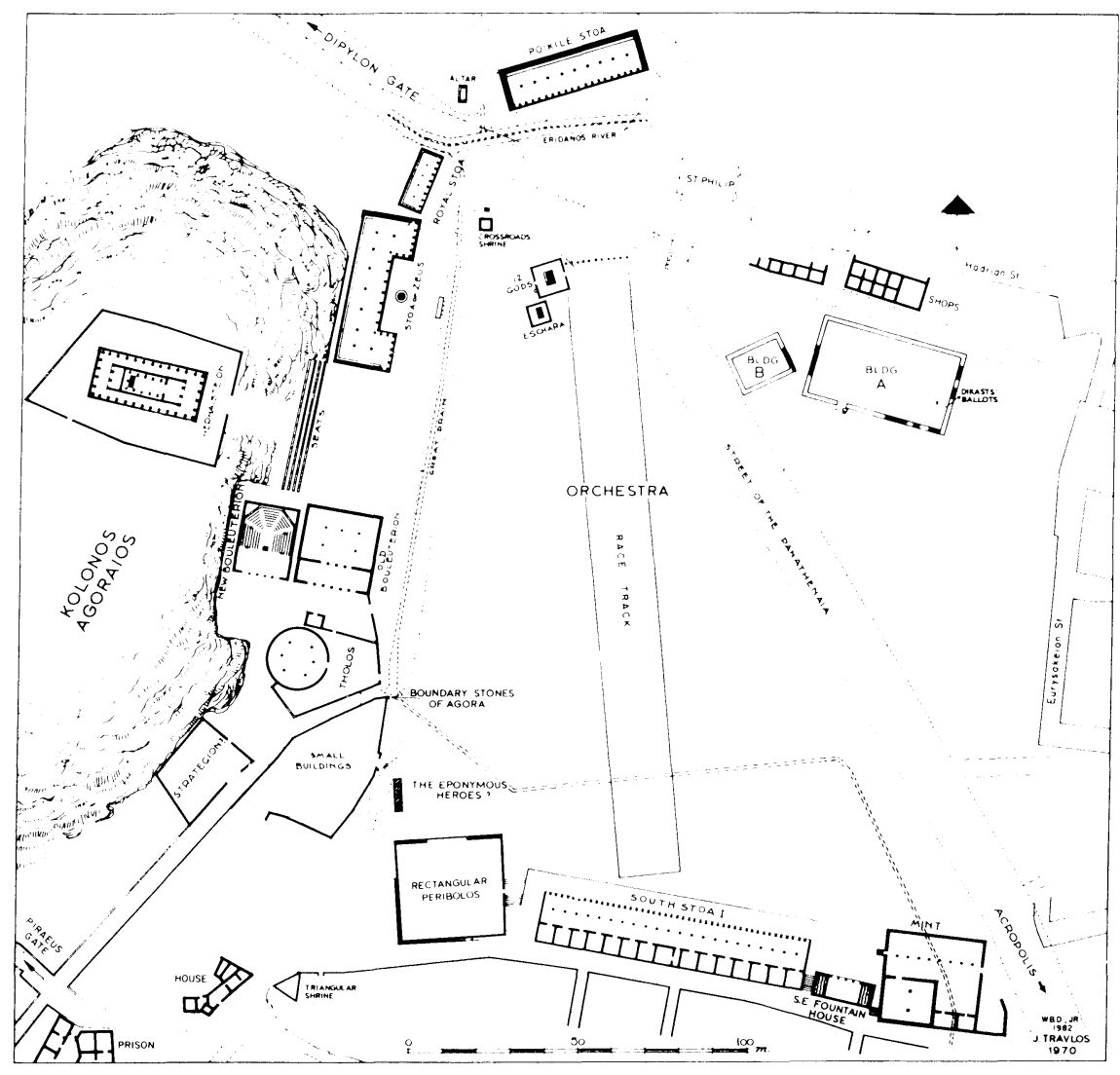

Fig. 2

Plan restitué de l'agora d'Athènes à la fin du ve siècle av. J.-C.

(D'après J. M. Camp, 1986 - voir bibliographie)

Cette proximité, voire cette confusion à l'intérieur des centres monumentaux entre l'espace des sanctuaires et l'espace de la place publique, s'explique par l'ampleur des zones réservées lors des premiers développements urbains. C'est en effet à l'intérieur de secteurs qui occupent souvent une part considérable de l'espace urbain que se définissent progressivement différentes zones. À Corinthe, le centre public de la ville occupe une vaste zone comprise entre le temple d'Apollon et même au-delà, la fontaine Glaukè et la série d'édifices qui sont situés au sud du dromos, c'est-à-dire sur toute l'étendue de la vaste cuvette qui s'étend jusqu'aux premières pentes de l'Acrocorinthe. À Poseidonia, l'espace laissé non bâti et qui fut occupé progressivement par les sanctuaires et l'agora représente une bande très allongée qui coupe littéralement la ville en deux : l'agora y trouve sa place entre le sanctuaire d'Athéna au nord et le sanctuaire d'Héra au sud. À Thasos, le centre monumental commence avec le Dionysion et l'Artémision, reliés par une grande place, et se termine à quelque deux cents mètres au sud.

L'affectation d'un secteur particulier des centres monumentaux aux activités politiques (réunion d'assemblées, siège de conseils ou de magistratures) se signale par la construction d'édifices destinés à les abriter. Nous avons évoqué plus haut les difficultés qu'il y avait à identifier chacun d'eux avec toute la précision souhaitée. Mais paradoxalement, les exemples les plus anciens sont ceux dont l'identification pose $a$ priori le moins de problèmes. C'est une fois encore vers les cités coloniales, dans ce domaine comme dans beaucoup d'autres certainement plus créatrices et peut-être plus précoces que les cités de vieille Grèce, qu'il faut se tourner.

L'agora de Métaponte offre en effet, à partir de la fin du VII siècle av. J.-C., très peu de temps après la fondation de la colonie, une extraordinaire succession de constructions destinées à accueillir une assemblée (fig. 1). La première consistait en une série de bancs en bois

7 HISTOIRE DE L'ART $\mathrm{N}^{\mathrm{O}} 42 / 43$ OCTOBRE 1998 
disposés sur une pente artificielle, dont il est malheureusement impossible de restituer le plan exact. C'est en tout cas la première attestation archéologique des ikria mentionnés par les sources littéraires, qui nous rapportent que ces premiers dispositifs furent abandonnés à la suite d'effondrements ou d'incendies catastrophiques : de fait, les gradins en bois de Métaponte ne doivent leur partielle conservation archéologique qu'à un incendie. À cette première phase encore modeste, succède au milieu du VI ${ }^{e}$ siècle av. J.-C. un édifice beaucoup plus ambitieux. Il s'agit d'une construction en pierre, de plan circulaire et constituée de deux caveae affrontées de part et d'autre d'une orchestra rectangulaire. Elle pouvait accueillir jusqu'à sept mille cinq cents ou huit mille personnes. Cet édifice à double hémicycle n'est pas unique, mais c'est le plus ancien. On trouve un édifice de plan identique à Agrigente et un autre, beaucoup plus petit, à Poseidonia : seulement cinq cent cinquante ou huit cents personnes (selon les restitutions) pouvaient y prendre place. Ces édifices étaient manifestement destinés à accueillir des assemblées, mais lesquelles? S'agit-il d'assemblées politiques, l'ekklesia à Métaponte et Agrigente, la boulè à Poseidonia? C'est probable, mais rien ne vient l'assurer avec certitude, si ce n'est leur emplacement sur l'agora : or celle-ci est précisément qualifiée de telle à partir de l'époque de leur construction. Ces édifices, avec leur koilon et leur orchestra, font penser aux théâtres. Au-delà de la parenté morphologique, est-ce le signe d'une parenté fonctionnelle? Précisons que tous les ekklesiasteria et tous les bouleuteria connus par ailleurs ne ressemblaient pas à ce plan. Même en présence d'édifices dont la restitution n'est pas controversée, les questions demeurent donc.

Quoi qu'il en soit, dans les agoras de la vieille Grèce, on ne retrouve pas de telles constructions à des époques aussi anciennes. Un édifice de ce genre a peut-être existé à Gortyne à la fin du VI ${ }^{e}$ siècle av. J.-C. mais, en règle générale, les agoras ne reçoivent leurs premiers édifices publics qu'à partir du début du Ve siècle, sauf exception comme à Siphnos, où l'agora et le prytanée reçoivent dès le VIe siècle une parure en marbre de Paros (Hérodote, III, 57, 4), réservée à cette époque aux édifices religieux, ou à Olympie, où le premier état du bouleuterion remonte à la deuxième moitié du VI ${ }^{e}$ siècle. Le cas d'Athènes est particulier (fig. 2). Il est en effet certain que l'espace sur lequel s'est développée l'agora classique était occupé encore par des nécropoles jusqu'à une date avancée du VI ${ }^{\mathrm{e}}$ siècle.

Si un centre civique était déjà constitué à cette date, il faut donc le chercher ailleurs, probablement au nord-est du rocher de l'Acropole, là où il convient sans doute de placer le prytanée de la cité. Le secteur compris entre l'Éridanos, la colline de Kolonos Agoraios et les premières pentes de l'Acropole, fut transformé en espace public avant tout pour accueillir des manifestations religieuses, aussi bien les fêtes lyriques des grandes Dionysies que les processions ou les concours lors des Panathénées : c'est ce dont témoignent les inscriptions mentionnant la piste de course (dromos), dont on a récemment mis au jour le dispositif de départ (de telles pistes ont été également retrouvées à Corinthe ou à Argos). Ce sont là les plus anciens témoignages certains d'une activité collective dans ce secteur. A cette époque, la place est encore un terrain vague : seule une fontaine au sud-est et une grande maison au sud-ouest attestent un début d'occupation monumentale. En revanche, rien n'indique une activité politique, ni même l'installation d'une agora. La première attestation d'une agora à cet endroit consiste en un message, inscrit sur des bornes placées chacune à l'angle formé par une rue et la place : " Je suis la borne de l'agora. " On s'accorde à dater ces inscriptions de la fin du VIe siècle. À partir de cette époque, de Clisthène à Cimon et Éphialte, ont lieu les premiers aménagements monumentaux du centre civique d'Athènes. Mais en dehors de la Stoa basileios, siège d'une très importante magistrature, ou de la tholos, qui servait de salle à manger aux prytanes, aucun édifice n'est spécialement construit pour accueillir un conseil, une assemblée, ou servir de siège à une magistrature. La restitution de l'édifice considéré depuis son dégagement comme le premier bouleuterion de la cité, est à juste titre remise en question : peut-être faut-il chercher le siège des bouleutes dans les gradins taillés à flanc de rocher sur les pentes du Kolonos Agoraios? Ces premiers aménagements dénotent en tout cas la polyvalence des activités qui se déroulaient sur ces vastes espaces : représentations théâtrales, concours et, progressivement, activités politiques.

Mais les centres civiques que deviennent alors les agoras n'eurent jamais l'exclusivité des fonctions politiques : si les prytanes de la cité d'Athènes prennent leur repas dans la tholos, le siège principal de leur collège - le prytanée proprement dit - se trouve ailleurs, et la construction d'un ekklesiasterion sur la Pnyx à la fin du Ve siècle entraîne le déplacement des assem- 
blées du peuple. Celles-ci pouvaient également se réunir au sanctuaire de Poséidon à Côlonos, à l'Anakeion sur les pentes de l'Acropole, au théâtre de Mounychie ou à celui de Dionysos. Dans les vieilles cités comme Athènes, beaucoup plus que la concentration des sièges de magistrats, des assemblées, des tribunaux ou des différents conseils, ce sont les investissements monumentaux sur ces places à partir de l'époque classique qui les font devenir un des pôles majeurs de l'organisation urbaine. Les structures architecturales dont elles sont dotées alors restent souvent polyvalentes. Le grand portique que fait construire Peisianax au nord de l'agora d'Athènes, la stoa Poikilè, était destiné à servir d'écrin à des tableaux, commandés aux peintres les plus illustres de l'époque, Micon et Polygnote de Thasos: mais il pouvait servir éventuellement de siège aux tribunaux. On sait par les textes, en particulier par la littérature judiciaire du IVe siècle, que certains tribunaux bénéficiaient d'un emplacement réservé sur l'agora; mais les identifications proposées jusqu'aujourd'hui, en particulier l'enclos retrouvé dans l'angle sud-ouest de la place, sont loin d'être certaines. Ces places ont parfois reçu des aménagements destinés au confort des badauds; par exemple, Cimon fait planter des platanes sur l'agora d'Athènes. Les colonnades des stoas pouvaient également fournir diverses commodités aux promeneurs... et aux philosophes qui, à force de s'y réunir, prirent le nom de "Stoïciens".

Dès cette époque, l'agora devient aussi un lieu de mémoire, où l'identité de la cité s'exprime avec force : le groupe des Tyrannoctones placé sur l'agora d'Athènes, immédiatement au bord de la voie des Panathénées, était censé perpétuer le souvenir de la chute des Tyrans, au prix d'ailleurs d'une manipulation historique. La qualité et le renom des artistes sollicités pour fabriquer les statues de culte des temples de l'agora, tel Euphranor, illustrent également la valeur que lui accordaient les Athéniens. Jusqu'à cette époque, l'agora reste le lieu de la convergence populaire au sens le plus large, c'est-à-dire un centre urbain qui pouvait accueillir les fêtes religieuses, les grands rassemblements politiques ou les marchés. D'Aristophane à Eubule, les auteurs attiques ne manquent pas d'évoquer le mélange sur ces places de toutes sortes de denrées et de personnages; à les lire, on pense plutôt aux bazars orientaux qu'à de beaux ordonnancements monumentaux... Ce n'est que plus tard, au IVe siècle et surtout au III $^{\mathrm{e}}$ siècle av. J.-C., d'abord dans des cités nouvelles ou refondées dont on prévoyait l'organisation dès le début, que l'agora se confondra avec le centre politique.

\section{La formation d'une place}

C'est à Kassopé que cette nouvelle organisation se laisse le mieux percevoir. Le plan de cette cité, fondée à la fin du Ve siècle av. J.-C., prévoit d'emblée, à l'intérieur de la trame formée par les îlots d'habitation, l'espace de l'agora (fig. 3). Mais sa bordure n'est que très progressivement bâtie. Dans sa phase la plus récente, le centre public est doté d'un édifice en forme de théâtre destiné à accueillir des assemblées, installé à l'ouest de la place (bouleuterion? ekklesiasterion?), et de deux portiques qui bordaient les côtés nord et ouest. Mais si cet aménagement trahit incontestablement un souci de concentration du centre politique dans la trame urbaine, l'agora n'est pas encore conçue comme un espace unique et centripète, intégrant l'ensemble des édifices civiques. En effet, c'est au-delà de la rue qui bordait l'arrière du portique nord, donc nettement en dehors de la place, que fut construite une vaste construction de plan rectangulaire, comportant dix-sept pièces réparties autour d'une cour péristyle (marché, édifice à banquets, hôtellerie ?). L'orientation de l'édifice d'assemblée est en outre désaxée par rapport à l'implantation de la place, sans doute pour utiliser au mieux la topographie. Il reste donc juxtaposé et isolé, comme le bouleuterion de Sicyone. Une nouveauté sera riche d'avenir : l'utilisation du portique comme façade de pièces ou d'édifices différents. Le portique ouest, en effet, est conçu pour donner une unité architecturale à ce côté de la place, précédemment occupé par différentes constructions. Cette formule sera retenue plus tard, lorsqu'il s'agira de donner une unité aux édifices de la bordure sud-ouest de l'agora d'Athènes (le Metrôon) ou pour servir de façade monumentale au prytanée et au bouleuterion de Priène.

Les agoras restent donc jusqu'à la haute époque hellénistique une forme urbanistique ouverte, dans laquelle l'espace central met en relation et unifie les différentes constructions, disposées sur les côtés en un assemblage assez flou. Si les différents éléments de la panoplie " classique " de l'agora ont déjà été élaborés, ils ne sont pas encore organisés de manière systématique. Le 


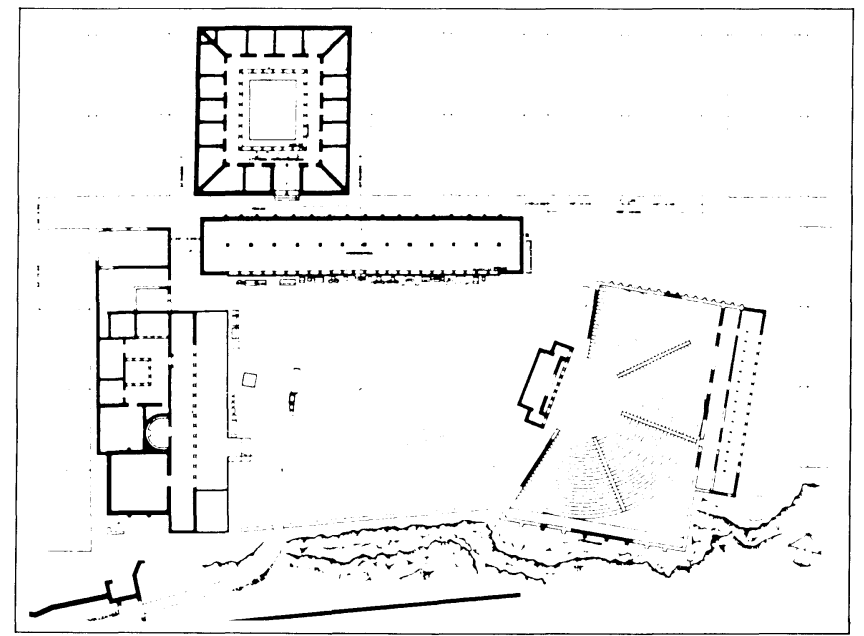

Fig. 3

Plan restitué de l'agora

de Cassopée au III" siècle av. J.-C.

(D'après W. Hoepfner et

E.-L. Schwandner, Haus und Stadt im

Klassischen Griechenland, Munich/

Berlin, 1994)

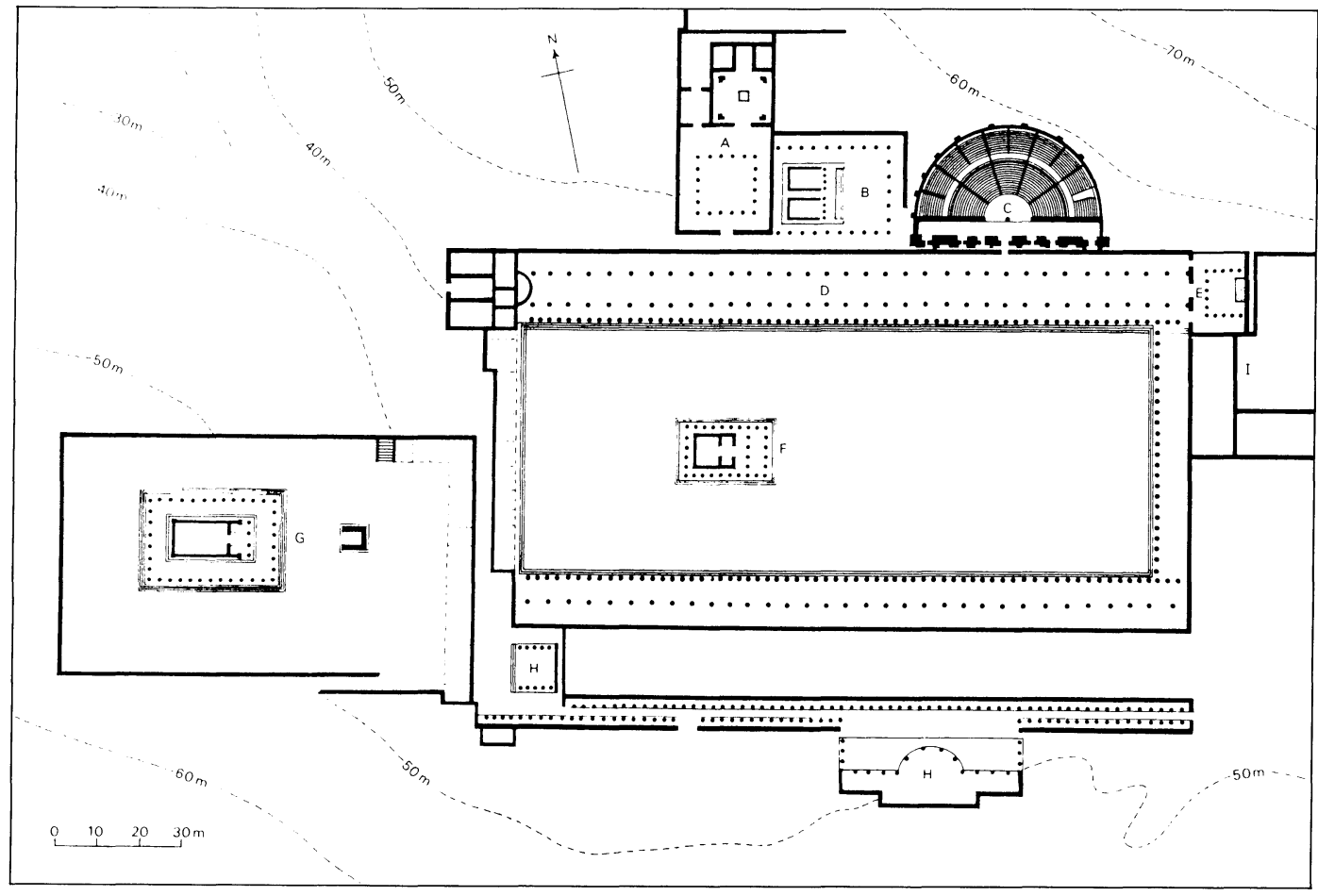

Fig. 4

Plan restitué de l'agora haute d'Éphèse au Ier siècle ap. J.-C.

(D'après W. Alzinger, 1974 - voir fig. 1) 
portique indépendant règne et les colonnades "écrans " ne sont utilisées que de manière ponctuelle. Comme la place elle-même, ces portiques sont des formes ouvertes, dans lesquelles on ne distingue pas très bien l'intérieur de l'extérieur, même si, dans certains cas, les entrecolonnements étaient fermés par des parois, comme la stoa nord-ouest de Thasos ou le portique du port aux lions de Milet. Cette forme architecturale, qui constitue à partir de cette époque l'élément le plus répandu des paysages urbains, permet d'abriter différentes activités (le commerce comme dans le portique d'Antiochos de l'agora sud de Milet, les archives comme dans la stoa nord-est de Mégalopolis). L'élasticité de son plan permet de l'allonger autant que nécessaire pour isoler la place de la voirie environnante, comme le portique est de l'agora sud de Milet $(189,2 \mathrm{~m})$, ou pour constituer une barrière monumentale sur tout un côté de l'agora comme le portique sacré de l'agora de Priène, le portique sud de l'agora de Corinthe, le portique de Philippe qui ferme le côté nord de l'agora de Mégalopolis (155,55 m).

Le progressif équipement architectural des agoras était tout autant destiné à jouer un rôle dans l'orgueilleuse affirmation des cités et des monarques donateurs, qu'à accueillir des fonctions précises. Souvent les constructions de l'époque hellénistique dépassent largement la surface nécessaire au bon fonctionnement de l'institution qu'elles étaient censées abriter : le bouleuterion de Milet par exemple, couvre une surface totale de $2100 \mathrm{~m}^{2}$ et pouvait accueillir au moins mille deux cents personnes, alors qu'il n'y eut jamais autant de bouleutes dans cette cité. Dès sa construction (deuxième moitié du II siècle av. J.-C.), cet édifice fut suivi en Asie Mineure par les bouleuteria d'Akrai, Alabanda, Aspendos, Iasos ou Priène, phénomène qui atteste la concurrence que se livraient les cités dans ce domaine, et la nécessité dans laquelle celles-ci se trouvaient de compléter leur équipement architectural. Par ailleurs, cette fièvre de constructions civiques en bordure des agoras hellénistiques ne manque pas de produire des effets, aussi bien sur l'urbanisme que sur la fonction des différents espaces qui se constituent progressivement à l'intérieur des centres monumentaux. Même si la formalisation définitive des espaces publics n'est réalisée qu'à une date assez tardive, le plus souvent au Ier siècle av. J.-C. (agora sud de Milet, agora de Thasos), le processus est en place, qui voit l'agora passer d'un espace libre, ouvert sur les rues adjacentes, à un espace construit. Ce phénomène accompagne une spécialisation progressive des différents secteurs ainsi délimités : les activités politiques et religieuses d'un côté, les activités commerciales d'un autre. Cette séparation, réclamée par Xénophon, Platon et surtout Aristote, qui préconise une distinction très nette entre une " agora libre " et une agora commerçante (Polit., 1331a), est à l'œuvre, dans certaines cités, à partir du IVe siècle av. J.-C. Ainsi à Thasos, la construction à peu de temps d'intervalle du portique nord-ouest et des magasins sud-est (fin $\mathrm{IV}^{\mathrm{e}}$ ou début $\mathrm{III}^{\mathrm{e}}$ siècle) révèle la volonté d'isoler le centre monumental du port et de la principale artère thasienne qui longeait l'agora au sud-est. De ce côté, la très longue série de cellules de deux pièces chacune, magasins ou ateliers, commandées uniquement par la rue et tournant littéralement le dos à la place publique, montre bien la volonté d'exclure les activités d'échange et de transformation du centre civique. Une inscription du dème de Sounion qui rapporte l'aménagement d'une nouvelle agora destinée expressément à rester un espace libre, alors que l'agora plus ancienne est occupée par des constructions, traduit peut-être la même évolution : un isolement des centres civiques rendant les places de plus en plus impropres aux foires qu'elles avaient pu accueillir par le passé. À Priène, à la fin du III ${ }^{\mathrm{e}}$ siècle av. J.-C., le centre civique est séparé du marché par l'aile ouest du portique en pi qui l'entoure. À cette époque se généralise sur les agoras l'exposition des stèles où sont inscrites les décisions de l'assemblée, en particulier les honneurs rendus aux citoyens les plus méritants, ou les listes de magistrats. Il n'y a naturellement pas de rupture brutale dans ce phénomène, qui voit les centres civiques se doter d'une spécificité à la fois urbanistique et fonctionnelle.

C'est le moment où l'agora, depuis longtemps déjà lieu et instrument de la concurrence des cités entre elles, est investie par les élites. Pour ces dernières, l'évergésie, motivée aussi bien par des préoccupations politiques ou sociales que par la fidélité à l'idéal civique, se traduit par le financement de petits monuments, soit des statues présentées sur des bases plus ou moins monumentales, soit des exèdres sur lesquelles prenaient place plusieurs effigies, de plus en plus prisées au fur et à mesure que s'affirment au grand jour les stratégies gentilices des grandes familles. Les édifices plus ambitieux comme les grands portiques peuvent être également financés par de simples particuliers, mais ils sont le plus souvent offerts par les rois hellénistiques, qui 
y voient un instrument " médiatique " particulièrement efficace, grâce à la gravure bien en vue d'une dédicace.

\section{La monumentalisation du centre civique}

La monumentalisation et la spécialisation, par rejet des activités jugées indignes, se généralisent à l'époque hellénistique tout en se renforçant l'une l'autre, mais ces deux phénomènes avancent à des rythmes différents selon les situations. Ils sont surtout attestés dans des cités où les programmes édilitaires pouvaient se déployer dans un espace urbain encore libre, non saturé par une dense occupation de l'espace ou contraint par une structure monumentale antérieure. C'est le cas de Magnésie du Méandre où l'agora, vaste rectangle de $188 \times 99 / 95 \mathrm{~m}$, est conçue d'emblée comme un quadriportique presque entièrement fermé et isolé de la voirie environnante. La place ainsi définie devient d'une certaine manière un monument en soi.

Ce mouvement de clôture des agoras se retrouve dans les anciennes cités de Grèce continentale ou des îles. Là, il se transforme, du fait de la nécessité de s'adapter à un plan masse qu'on ne peut modifier. La création de places fermées doit passer soit par la régularisation des bordures au moyen de grands portiques, comme à Thasos, soit par la construction à proximité immédiate d'un nouveau complexe monumental, comme à Messène où, au sud de l'agora, est aménagé un quadriportique, comportant au centre de la cour un temple dédié au dieu tutélaire des Messéniens, Asklépios, et, dans les pièces abritées par les portiques, d'autres lieux de culte ou des espaces destinés à accueillir des activités spécifiquement politiques. En effet, on trouve successivement dans le portique ouest un bouleuterion et une salle de réunion. Il ne s'agit sans doute pas de dédoubler le vieux centre civique, le lien avec celui-ci étant fortement exprimé par un passage monumental, mais plutôt de satisfaire au goût des places closes en réservant à côté du centre civique traditionnel une "agora sacrée ". Mais ces deux possibilités, offertes aux édiles des vieilles cités, déjà densément bâties, ne sont pas partout réalisables.

À Athènes, où il n'était pas possible de condamner, ni même de détourner la voie des Panathénées qui traversait l'agora en diagonale avant de rejoindre les Propylées de l'Acropole, on dut se contenter d'une systématisation incomplète. Au milieu du II ${ }^{\mathrm{e}}$ siècle av. J.-C., trois constructions nouvelles donnent à la place sa forme définitive : sur la bordure est, un portique à étage que le roi de Pergame Attale II (159-138 av. J.-C.) offre à la cité; sur le côté sud, un grand complexe constitué de deux portiques de part et d'autre d'une cour; sur le côté ouest, une reconstruction complète du Metrôon et de la salle des archives, réunis par une colonnade ionique. Si désormais des colonnades marquent sur tous les côtés les limites de la place, cellesci ne sont pas continues; les différentes constructions restent isolées les unes des autres, leur disposition trahit l'ancienneté de l'occupation de l'espace et, sauf pour la stoa d'Attale et le complexe sud, l'absence de tout plan d'urbanisme. De ce point de vue, l'agora d'Athènes illustre parfaitement l'agora de type ancien, telle que la définit Pausanias (VI, 24, 2). À ce type, il oppose les agoras "ioniennes ", terme qui désigne certainement les grands quadriportiques des cités micrasiatiques (voir aussi Vitruve, V 1,1). Ceux-ci ne sont d'ailleurs pas réservés aux centres civiques. Il s'agit d'une formule architecturale née de la transposition des péristyles de l'architecture domestique à des espaces beaucoup plus vastes.

S'ils caractérisent aux yeux de Pausanias les agoras des grandes villes de la côte ionienne, les exemples les plus anciens se trouvent dans les résidences macédoniennes. On peut d'ailleurs se demander si l'agora hellénistique de Pella, actuellement en cours de dégagement, ne reprend pas dans ses grandes lignes le plan de ces dernières. Signalons d'ailleurs qu'à Pella, capitale royale, l'agora semble n'avoir d'autres fonctions que commerciales. Toujours est-il que ce type de plan se répand d'abord dans les palestres ou les sanctuaires, avant d'être adopté pour les centres civiques. À la fin de l'époque hellénistique, la vogue de cette formule aboutit à son application répétitive dans tous les espaces monumentaux. A Pergame, gymnases, sanctuaires, palais ou agora présentent à peu près le même plan. Le bouleuterion de Milet possède sa propre cour à colonnades. À Délos, malgré le nombre de places déjà constituées — le sanctuaire d'Apollon, l'agora tétragone ainsi que les dégagements plus informels de la façade maritime : l' "agora de Théophrastos " et l' "agora des Compétaliastes " — on décide de satisfaire à cette mode des places péristyles isolées en construisant l'agora des Italiens, sans doute pour

12 LES AGORAS GRECQUES D'APRÈs LES RECHERCHES RÉCENTES 
y accueillir les activités qui ne pouvaient plus ou pas l'être ailleurs, un peu à la manière dont on construira à Athènes l' "agora romaine ".

La banalisation architecturale des agoras s'accompagne, à la fin de l'époque hellénistique, d'un détournement des investissements monumentaux vers d'autres lieux, en particulier le gymnase. Ces nouveaux édifices, quand ils ne sont pas construits directement sur l'agora comme à Argos, ou à côté comme à Mégalopolis, constituent de nouveaux centres attractifs dans la trame urbaine. Devenus des lieux importants de la vie sociale, investis rapidement par les notables, ils forment en quelque sorte, selon le mot de L. Robert, une "seconde agora".

L'entrée progressive de l'Orient méditerranéen dans l'orbite politique de Rome n'entraine pas de changements brutaux dans les agoras des vieilles cités grecques. Il y a plusieurs raisons à cette étonnante continuité. La première tient à l'influence ancienne et importante des agoras grecques sur les réalisations monumentales de Rome, du Latium ou de la Campanie à l'époque républicaine. On connaît le rôle des architectes grecs dans les grandes réalisations de l'Urbs dès le III siècle av. J.-C., et la parenté entre les complexes architecturaux du Champ de Mars et les programmes édilitaires des grandes villes hellénistiques. Même des constructions spécifiquement romaines, comme les comitia, passaient aux yeux des contemporains pour dériver de prototype grecs, les ekklesiasteria/bouleuteria (Denys d'Halicarnasse, 4, 38). À Poseidonia devenue la colonie romaine de Paestum, malgré la volonté de rupture dont témoigne le déplacement du centre civique (le forum est légèrement plus au sud que l'ancienne agora), le siège des comices reprend presque purement et simplement le plan de l'édifice d'assemblée archaïque.

La deuxième raison tient au fait qu'à l'inverse des noyaux urbains du monde occidental, les villes grecques, et les agoras en particulier, étaient déjà entièrement équipées de monuments que les Romains considéraient comme les symboles de la dignitas urbaine. Par ailleurs, les nécessités de l'administration provinciale n'impliquaient qu'exceptionnellement des constructions nouvelles. Pas plus que jadis les institutions civiques, les organes provinciaux n'avaient besoin d'installations spécifiques. À Mégalopolis, une simple tribune construite dans l'axe de la nef centrale du portique de Philippe, contre le mur du petit côté est, suffit pour servir de locus superior, et permettre au préteur d'y rendre la justice : ce simple aménagement transformait le portique hellénistique en basilique romaine. Même quand une véritable basilique vient compléter l'ensemble des monuments d'une agora grecque, la parenté entre sa forme et le portique de tradition hellénistique fait qu'il n'y a pas non plus dans ce cas de changement dans le plan masse de la place, susceptible d'être interprété en terme de romanisation. Parfois même, l'espace basilical proprement dit est disposé à l'arrière d'une colonnade de façade qui l'intègre aux édifices déjà existants; c'est le cas, à Thasos, du complexe formé par la grande galerie hypostyle et le portique coudé.

Le cas d'Athènes, trop souvent érigé en exemple, passe pour l'illustration de la réduction des cités grecques au rôle de villes-musées, condamnées en quelque sorte à une vieillesse heureuse, mais dépendante et assistée. Il est vrai que les différentes constructions du tout début de l'époque impériale ont fondamentalement modifié l'aspect de la place. L'odéon, offert par Agrippa entre 16 et 12 av. J.-C., le déplacement à la même époque d'un temple et d'un autel d'Arès, entièrement démontés à Acharnes pour être placés en face du temple d'Apollon Patrôos, la reconstruction de temples, littéralement spoliés à Sounion ou Thorikos pour figurer respectivement dans l'angle sud-ouest et à l'entrée sud-est de l'agora, aboutissent à une considérable densification de l'espace bâti au détriment de la place elle-même. Mais faut-il y voir l'instrument d'un anéantissement brutal du rôle civique et politique de l'agora? Si l'espace central est en effet rendu inutilisable pour réunir l'assemblée, servait-il encore à cet usage à l'époque ? Il est une nouveauté qui mérite d'être relevée : l'apparition d'une dimension verticale dans cet espace, caractérisé jusqu'alors par l'horizontalité et les longues perspectives rythmiques des colonnades des portiques. L'odéon d'Agrippa, dont la hauteur est plus du double de celle du portique sud, devait imposer sa masse à la manière d'un temple dans un sanctuaire. Pour le reste, les changements s'inscrivent dans la continuité d'une transformation déjà ancienne : la spécialisation de plus en plus nette des différents secteurs du centre monumental. Les transformations de l'agora correspondent à une politique édilitaire qui concernait l'ensemble de la zone publique au nord de l'Acropole et de l'Aréopage. C'est alors que le vieux centre d'Athènes s'enrichit de l' "agora romaine ", destinée à accueillir des activités commerciales. À l'époque d'Hadrien (117-138 ap. J.-C.), un nouveau complexe monumental, la " biblio-

13 HISTOIRE, DE, L.ART N $\mathrm{N}^{0} 42 / 43$ OC:TOBRE 1998 
thèque d'Hadrien ", sera bâti non loin au nord. On a récemment remarqué que son plan reprend exactement celui du forum de la Paix à Rome: il s'agit vraisemblablement encore d'une "agora ", destinée à abriter des activités administratives. Cet isolement monumental, qui n'est pas un fait nouveau, se manifeste ailleurs par la construction de véritables propylées (Magnésie du Méandre ou agora sud de Milet à l'époque antonine).

Les constructions ou reconstructions d'agoras dans les cités grecques à l'époque impériale montrent que le type même du centre civique monumental, qu'elles étaient devenues à l'époque hellénistique, avait gardé toute sa valeur. À l'époque augustéenne, une agora est entièrement rebâtie à Éphèse à l'extrémité est de la rue des Courètes (fig. 4). Elle comporte les nouveautés architecturales de l'époque, comme la basilique, et des édifices plus traditionnels - prytanée et bouleuterion -, l'ensemble étant construit à l'arrière d'un quadriportique centralisateur, selon la mode en vigueur depuis l'agora de Magnésie du Méandre. À la même époque, le forum de la colonie romaine de Corinthe s'articule sur la reconstruction du portique qui bordait au sud l'agora de la cité grecque avant sa destruction: la continuité avec la disposition des édifices classiques et hellénistiques ne s'explique pas uniquement par les contraintes topographiques.

Quand au culte impérial, signe et instrument de la domination politique de Rome, il n'est pas toujours aussi imposant qu'on l'a dit : il convient en tout cas de distinguer entre les grandes capitales provinciales (Éphèse, Corinthe) où le sanctuaire de l'empereur domine la place publique, et les cités moyennes où il prend la suite des cultes hérö̈ques et civiques, faminiliers des agoras grecques. Celles-ci restent donc, plus longtemps que les fora occidentaux, le lieu de l'identité historique de la cité et le symbole de l'autonomie municipale. Certes, les dédicaces de statues d'empereurs deviennent de plus en plus nombreuses à partir de l'époque antonine, mais les riches familles locales ne délaissent pas pour autant ce lieu prestigieux : elles continuent d'y faire installer leurs effigies. Le rôle politique de ces centres ne s'est pas évanoui, il s'est transformé.

Pausanias, une des sources les plus riches sur les agoras d'époque impériale, les décrit en présentant ce qu'il voit - édifices civiques, sanctuaires, offrandes, dédicaces - comme autant de monuments historiques, dignes par conséquent d'être admirés, mais illustrant une époque révolue. Mais Pausanias était un antiquaire, féru d'une culture passéiste, comme d'ailleurs ses contemporains de la seconde sophistique. Les sources épigraphiques donnent des centres civiques une image différente et attestent l'attachement des citoyens à leur agora. Les élites continuent de tenir à jour les listes de magistrats et de les exposer dans la place publique, comme à Thasos, et le peuple de s'y exprimer, comme à Magnésie du Méandre où des boulangers se voient interdire l'accès de l'agora en groupe pour y avoir précédemment exprimé leur colère.

Entretenues jusque dans le courant du IV siècle, les agoras disparaissent avec la christianisation des villes et l'apparition de nouveaux pôles monumentaux, comme les églises.

Les recherches récentes ont fait perdre au concept moderne d'agora la trompeuse clarté qu'il avait pu avoir. Elles ont montré que les agoras avaient une spécificité moins nette et moins ancienne qu'on n'avait cru, qu'elles ne se confondaient pas d'emblée avec le centre politique, qu'elles ne concentraient pas partout l'ensemble des édifices civiques, qu'à peine leur caractère architectural et fonctionnel mis au point, elles s'intégraient dans un ensemble de complexes monumentaux, qui les dépassait largement. L'attention a été attirée sur des questions, des sources ou des régions jusque-là négligées. Mais surtout, il convient d'abandonner tout discours systématique. Car les agoras grecques présentent une très grande diversité selon les lieux, les époques, la situation respective des villes considérées. Leur évolution est loin d'être linéaire. Comment comparer l'agora, ou plutôt l'une des agoras, d'un grand centre portuaire comme Milet avec celle d'une petite cité à l'écart des modes et surtout privée de moyens, comme Lato? Un centre monumental ancien investi de toutes les valeurs d'une tradition séculaire comme Athènes avec une fondation éloignée de plusieurs milliers de kilomètres du bassin de l'hellénisme comme Aï-Khanoum? L'agora d'une cité libre et autonome comme Thasos avec une capitale royale comme Pergame? À l'inverse du " bloc forum " romain, qui associe dans une formule architecturale répétitive le culte capitolin, le culte impérial et les principaux or-

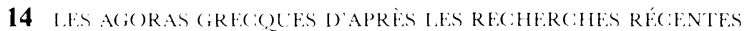


ganes de gouvernement et d'administration, l'agora grecque est restée tout au long de son histoire à l'image de la cité grecque : chatoyante, changeante, contradictoire..., insaisissable.

Jean-Yves MARC:

Université de Strasbourg II

\section{INDICATIONS BIBLIOGRAPHIQUES}

La synthèse de R. Martin, Recherches sur l'agora grecque. Études d'histoire et d'architecture urbaine, Paris, de Boccard, 1951, reste l'étude la plus synthétique et la mieux documentée pour les sources littéraires. Pour une mise à jour archéologique, on consultera le bulletin bibliographique publié régulièrement par la Revue archéologique aux rubriques : " architecture civique ", " urbanisme " et " lieu de rencontre ". Parmi les études les plus récentes, on retiendra :

- F. Kolb, Agora und Theater, Volks- und Festversammlung, Berlin, Gebr. Mann Verlag, 1981.

- P. Fraisse, "Analyse d'espaces urbains : les places à Délos ", Bulletin de correspondance hellénique, 107, 1983, pp. 301-313

- F. Felten, "Heiligtümer oder Märkte?", Antike Kunst, 26, 1983, pp. 84-105.

- J. M. Camp, The Athenian Agora. Excavations in the Heart of Classical Athens, New York, Thames and Hudson, 1986.

- W. Koenigs, "Planung und Ausbau der Agora von Priene ", Istanbuler Mitteilungen, 43, 1993, pp. 381397.

- M. H. Hansen et T. Fischer-Hansen, "Monumental Political Architecture in Archaic and Classical Greek Poleis. Evidence and Historical Significance ", dans D. Whitehead (ed.), From Political Architecture to Stephanus Byzantius. Sources for the Ancient Greek Polis, Stuttgart, F. Steiner, 1994, pp. 23-90.

- S. Miller, "Old Metroon and Old Bouleuterion in the Classical Agora of Athens ", dans M. H. Hansen et K. Raaflaub (ed.), Studies in the Ancient Greek Polis, Stuttgart, F. Steiner, 1995, pp. 133-156

- J.-Y. Marc, "L'agora de Thasos ", dans L'espace grec. 150 ans de fouilles de l'École française d'Athènes, Paris, Fayard, 1996, pp. 105-113.

- P. Marchetti et K. Kolokotsas, Le nymphée de l'agora d'Argos. Fouille, étude architecturale et historique (Études péloponésiennes XI), Paris, de Boccard, 1995.

- D. Mertens, "Metapont. Ein neuer Plan des Stadzentrums ", Archäologischer Anzeiger, 1985, pp. 645-671.

- P. Gros, "Les nouveaux espaces civiques du début de l'Empire en Asie Mineure : les exemples d'Éphèse,

Iasos et Aphrodisias ", Aphrodisias Papers, 3 (Journal of Roman Archeology Supplement, 20), New York, Ann Arbor, 1996, pp. 111-120. 